\title{
Dense Plasma Focus: A question in search of answers, a technology in search of applications
}

\author{
S. K. H. Auluck \\ Physics Group, Bhabha Atomic Research Center, Mumbai 400085, India \\ skauluck@barc.gov.in,skhauluck@gmail.com
}

Published 13 August 2014

\begin{abstract}
Diagnostic information accumulated over four decades of research suggests a directionality of toroidal motion for energetic ions responsible for fusion neutron production in the Dense Plasma Focus (DPF) and existence of an axial component of magnetic field even under conditions of azimuthal symmetry. This is at variance with the traditional view of Dense Plasma Focus as a purely irrotational compressive flow. The difficulty in understanding the experimental situation from a theoretical standpoint arises from polarity of the observed solenoidal state: three independent experiments confirm existence of a fixed polarity of the axial magnetic field or related azimuthal current. Since the equations governing plasma dynamics do not have a built-in direction, the fixed polarity must be related with initial conditions: the plasma dynamics must interact with an external physical vector in order to generate a solenoidal state of fixed polarity. Only four such external physical vectors can be identified: the earth's magnetic field, earth's angular momentum, direction of current flow and the direction of the plasma accelerator. How interaction of plasma dynamics with these fields can generate observed solenoidal state is a question still in search of answers; this paper outlines one possible answer. The importance of this question goes beyond scientific curiosity into technological uses of the energetic ions and the high-power-density plasma environment. However, commercial utilization of such technologies faces reliability concerns, which can be met only by first-principles integrated design of globallyoptimized industrial-quality DPF hardware. Issues involved in the emergence of the Dense Plasma Focus as a technology platform for commercial applications in the not-too-distant future are discussed.
\end{abstract}

Keywords: Dense Plasma Focus; ion beams; capacitors; numerical simulation.

\section{Introduction}

Neutron emission from the Dense Plasma Focus ${ }^{1}$ (DPF) is known to possess several features which are not fully explained till date. It is about two orders of magnitude larger than estimates ${ }^{2}$ based on a Maxwellian ion velocity distribution with measured plasma parameters. Diagnostics based on D-D fusion product spectroscopy reveal the following features of the deuteron population participating in the fusion reaction:

- Neutron time-of-flight (ToF) spectra measured axially in the direction of current flow $\left(0^{\circ}\right)$ and in the opposite direction $\left(180^{\circ}\right)$ consistently show shift of the spectral peak at $2.45 \mathrm{MeV}$ to higher and lower energies ${ }^{3-6}$ respectively.

This is an Open Access article published by World Scientific Publishing Company. It is distributed under the terms of the Creative Commons Attribution 3.0 (CC-BY) License. Further distribution of this work is permitted, provided the original work is properly cited. 
- The obvious inference that this implies the presence of a linear ion beam with kinetic energy of the order of $10^{5} \mathrm{eV}$ moving in the direction of current flow is belied by unmistakable signature of accelerated deuterons in the opposite direction as well ${ }^{7-9}$.

- Neutron ToF spectrum on the Frascati $1 \mathrm{MJ}$ plasma focus at $90^{\circ}$ to the axis with a 128 m flight path ${ }^{10}$ showed 3 peaks: one central peak centered on the $2.45 \mathrm{MeV}$ Q-value of the D-D fusion reaction with a Full Width at Half Maximum (FWHM) of 0.2-0.5 MeV and two distinct side peaks. According to the authors ${ }^{10}$, such peaks were consistent with "a $100 \mathrm{keV}$ deuteron loop in the plane determined by the gun axis and the observational direction". Sometimes, these peaks were not sufficiently separated resulting in a single broad peak ${ }^{10}$ with a width in the range of 0.5-0.7 MeV, which have been observed on other installations as well ${ }^{11,12}$.

- Space resolved time-integrated neutron spectra from the Frsacati 1 MJ plasma focus, measured in single shots by nuclear emulsion plate method, using 4 collimators looking to the left and right of the axis from two opposite sides, showed ${ }^{13}$ "evidence of a $100 \mathrm{keV}$ $\mathrm{d}+$ stream orbiting in a plane perpendicular to the experiment main axis". Space-resolved single-shot time-integrated fusion proton spectra from laterally mirror-symmetric locations, measured on the POSEIDON plasma focus also show ${ }^{14}$ asymmetry about the reaction Q-value, suggestive of a rotational motion of center-of-mass of reacting ions.

These features, including the lateral asymmetry of fusion reaction product spectra, can be reproduced by assuming that the energetic ions have a toroidal trajectory ${ }^{15}$. It is however difficult to account for the laterality (handedness) of the azimuthal motion in terms of a physical mechanism, for reasons which are described later.

Significantly, there are observations of an axial component of magnetic field in the plasma focus, even under conditions of azimuthal symmetry. The first evidence concerning presence of such axial magnetic field component came from an experiment at the Lebedev Physics Institute ${ }^{17}$ in which a laser pulse evaporated the copper anode of the Filippov type plasma focus while the plasma current sheath (PCS) was still some distance away from the axis. The copper plasma plume was found (using 5 frame pulsed ruby laser interferometry and time integrated soft $\mathrm{x}$-ray pinhole photography) to have negligible radial expansion, indicating presence of a magnetic field responsible for radial confinement. In a similar experiment ${ }^{18}$, the plasma plume was generated at an off-axis location and was seen to be pushed towards the axis and then to expand axially with negligible radial expansion. Since the current supplied by the capacitor bank and carried in the PCS had still not reached the axis, this was interpreted as indicating presence of an axial magnetic field component produced by the imploding plasma current sheath.

Axial magnetic field was detected in the Frascati 1-MJ plasma focus ${ }^{13,19}$ by Faraday rotation and in POSEIDON plasma focus at Stuttgart by reconstruction of fusion reaction proton trajectories ${ }^{16}$. A diamagnetic loop, perpendicular to and centered with the axis and placed outside the cathode of a small plasma focus at a distance $1 \mathrm{~cm}$ above the anode consistently detected a signal representing rate of change of axial flux ${ }^{20}$. Magnetic probes with transverse component rejection ratio of 30:1 detected distinct signals representing rate of change of radial, axial and azimuthal components of magnetic field in the 
POSEIDON plasma focus ${ }^{21}$ at a radial distance $80 \mathrm{~mm}$ from the axis and $8 \mathrm{~mm}$ above the anode surface. Magnetic probe measurements ${ }^{22}$ by Krauz et al. have confirmed the existence of spontaneous axial magnetic field component in the PF-1000 plasma focus device. Kubes et al. present data ${ }^{23,24}$ showing that neutron emission in PF-1000 is correlated with the increase and decrease of the spontaneous axial magnetic field.

Krauz et al. designed ${ }^{22}$ a probe which could simultaneously measure axial and azimuthal components of magnetic field and optical emission from the plasma from its radial position. Their observations reveal a previously unreported characteristic structure in the axial magnetic field signal. The axial magnetic field signal has two parts: the larger part coincides with the azimuthal magnetic field signal, which comes from the plasma current sheath (PCS). Correlations ${ }^{23}$ between interferometry and magnetic probe measurements show that this current carrying layer, about $1.6-2.6 \mathrm{~cm}$ in thickness, is located behind the dense plasma layer. There is a smaller pre-pulse of axial magnetic field, which has polarity opposite to the main body of the axial magnetic field and which is co-located with a $0.5 \mathrm{~cm}$ thick low density region $\left(<10^{24} \mathrm{~m}^{-3}\right)$ downstream of the dense plasma sheath seen in the interferograms, which is current-free and luminous according to the probe measurements. The reversal of the axial magnetic field signal coincides with the start of the azimuthal magnetic field signal. The pre-pulse axial magnetic field is directed along the outward normal to the anode of PF-1000, which shall be referred as $+z$ direction; the main body of axial magnetic field signal is therefore directed along the $-z$ direction.

The existence of spontaneous axial magnetic field in the dense plasma focus throws in sharp relief the problem about laterality inferred from space-resolved fusion product spectroscopy data. The measurements ${ }^{22}$ of Krauz et al. reveal a fixed polarity for the axial magnetic field signal; reports from Frascati ${ }^{13}$ and Stuttgart ${ }^{16}$ also confirm the existence of a fixed polarity. Since the equations of plasma dynamics neither contain a preferred direction nor contain any lateral asymmetry, the existence of a fixed polarity of axial magnetic field and lateral asymmetry of ion motion must be explained in terms of initial conditions, or equivalently, in terms of interaction of the plasma with an external physical vector field.

Only four such external vector fields are identifiable: earth's magnetic field, earth's angular momentum, direction of current density and direction of the plasma accelerator (from breech towards muzzle of the coaxial gun). It is difficult to imagine how the last three vector fields might interact with plasma dynamics to produce an axial magnetic field. Compression of earth's magnetic field to kilogauss level by the highly conducting plasma has already been suggested ${ }^{17,22}$ as an explanation of the observed axial magnetic field; however, this explanation is problematic as discussed below. How interaction of plasma dynamics with these fields can generate the observed solenoidal state is a question still in search of answers.

Amplification of magnetic field by current induced in a moving conductor always preserves the direction of the initial magnetic field. Reference ${ }^{25}$ to International Geomagnetic Reference Field IGRF-11 and satellite map of the building housing PF- 
1000 installation reveals that the component of earth's magnetic field along the outward normal to the PF-1000 anode face is $+16400 \mathrm{nT}$. This means that the weaker magnetic field in the pre-pulse region, co-located with the relatively cold precursor plasma ahead of the current carrying hot plasma, is along the external magnetic field; the much higher axial magnetic field in the hot plasma zone, which corresponds to a conductor moving in a pre-existing magnetic field, has polarity opposite to the external magnetic field.

The significance of the newly discovered axial magnetic field pre-pulse in the PF1000 measurements $^{22}$ is that it is located in a current-free, low-density, weakly ionized plasma situated in the transition zone between the neutral gas (which has no optical emission) and a fully-ionized, highly conducting, current-carrying plasma. Mechanisms based on magnetohydrodynamic phenomena, including the dynamo effect, therefore cannot be used to explain its origin or properties.

This observation casts doubt on dynamo-type ambient magnetic field amplification as a candidate mechanism responsible for axial magnetic field generation and suggests an alternate mechanism, which is discussed in section 2. Section 3 examines implications of the suggested mechanism for commercial utilization of DPF-based technologies and possibilities for its experimental validation /refutation.Optimism concerning commercial potential of the DPF has to face up to the reliability limitations of currently home-made hardware.Emergence of DPF as a technology platform for commercial applications is going to depend critically on development of industrial-quality hardware. Issues concerning such developments are discussed in section 4 .

\section{Generation of axial magnetic field by paramagnetic drift currents}

An average ion or electron in the weakly ionized current-free plasma region ahead of the PCS, which is referred here asprecursor plasma (PP), would be moving along the $-\hat{r}$ direction and would experience earth's magnetic field in the $+\hat{z}$ direction; the resulting Lorentz force would be in the $+\hat{\theta}$ direction for ions and $-\hat{\theta}$ direction for electrons. The associated azimuthal current would clearly beparamagnetic: the magnetic field produced by the current would be along the external magnetic field. This should lead to amplification of the external magneticfield. This is different from a dynamoeffect. The dynamo mechanism operates in plasmas which have a high conductivity by virtue of a low collision frequency caused by high electron temperature; the higher conductivity produces a higher current for a given electric field produced within the conducting medium because of its motion in a pre-existing magnetic field, in turn producing a greater reinforcement of the pre-existing magnetic field.

In contrast, the azimuthal drift of individual particles referred above is independent of collision frequency; it depends only on theexistence of free charged particles. The PP region is the transition zone between the neutral fill gas and the fully ionized PCS, where the degree of ionization varies from zero to one and the plasma density varies from zero to $10^{24} \mathrm{~m}^{-3}$ over a scale length of few millimeters. Therefore, characteristic scale lengths such as the Debye length, collisionless ion skin depth $c / \omega_{p i}$, collisionless electron skin depth $c / \omega_{p e}$ themselves vary over orders of magnitude, both much larger and smaller 
than the physical scale length of the precursor plasma region.Consequently phenomena like deviations from quasi-neutrality, Hall Effect and electron inertia ${ }^{26}$ cannot be neglected ab initio in any continuum model applied to this plasma region. Analysis of charged particle drift based on continuum models is therefore problematic.

In the absence of fundamental studies ${ }^{27,28}$ related to the physical regime of the precursor plasma region, the azimuthal drift of individual charged particles in a weakly ionized azimuthally symmetric, current-free imploding plasma placed in an axial magnetic field can be calculated ${ }^{29}$ without reference to any continuum approximation for the plasma as follows. The motion of a charged particle, of charge $q$ and mass $m$, acted upon by an average radially-directed force $-F_{r} \hat{r} ; F_{r}>0$, in an azimuthally symmetric axial magnetic field $B_{z e x t}$ in the $(x, y)$ plane is described by the equations

$$
\begin{aligned}
& m \ddot{r}-m r \dot{\theta}^{2}=q r \dot{\theta} B_{z e x t}-F_{r} \\
& m r^{2} \dot{\theta}+q r A_{\theta}(r)=\ell
\end{aligned}
$$

Here $A_{\theta}(r)$ is the vector potential and $\ell$ is the canonical momentum, which is conserved for the orbit. Eliminating $\dot{\theta}$, equation (1) yields

$$
m \ddot{r}=+\frac{\left(\ell-q r A_{\theta}(r)\right)^{2}}{m r^{3}}+q B_{z e x t} \frac{\left(\ell-q r A_{\theta}(r)\right)}{m r^{2}}-F_{r}
$$

For sufficiently small values of $r$, the first positive definite term dominates, so that the particle accelerates away from the axis; for sufficiently large values of $r$, the first term becomes smaller than the third negative definite term so that the particle accelerates towards the axis. Equation (2) is therefore formally identical with the equation for the oscillations of a particle in a one-dimensional potential well $V(r)$,

$$
m \ddot{r}=-V^{\prime}(r)
$$

whose solutions can be written as $r(t)=r_{0}+r_{1}(t)$, where $r_{0}$ is the coordinate of the bottom of the potential well, $V^{\prime}\left(r_{0}\right)=0$, and $r_{1}(t)$ is its oscillating component. The average azimuthal drift velocity is given by

$$
v_{\theta, d r i f t} \equiv\langle r \dot{\theta}\rangle=\lim _{T \rightarrow \infty} \frac{1}{T} \int_{0}^{T} \frac{\left(\ell-q r(t) A_{\theta}(r(t))\right)}{m r(t)} d t
$$

The integrand in (4) can be expanded in a Taylor series about $r_{0}$ and to first order in $r_{1}(t)$,

$$
\left.v_{\theta, \text { drift }} \cong \frac{\left(\ell-q r A_{\theta}(r)\right)}{m r}\right|_{r=r_{0}}+\left.\frac{\partial}{\partial r} \frac{\left(\ell-q r A_{\theta}(r)\right)}{m r}\right|_{r=r_{0}} \lim _{T \rightarrow \infty} \frac{1}{T} \int_{0}^{T} r_{1}(t) d t
$$


Since $r_{1}(t)$ is a bounded oscillating function, the second term is zero. Since $r_{0}$ is a solution of $V^{\prime}(r)=0,(5)$ becomes

$$
v_{\theta, \text { drift }} \cong \frac{q r_{0}}{2 m}\left[ \pm \sqrt{B_{z e x t}^{2}+\frac{4 m F_{r}}{r q^{2}}}-B_{z e x t}\right]_{r_{0}}
$$

Note that if the average force per particle is not uniformly pointing in the $-\hat{r}$ direction, (2)cannot be represented by a potential well with a minimum and the motion of the particle cannot be described in terms of a bounded oscillating function, resulting in collapse of the argument leading to (6).

Eq. (6) would be invariant under the transformation $\hat{z} \rightarrow-\hat{z}$ only if the sign of the square-root is related to the sign of the $z$-component of a physical vector. For $B_{z e x t}^{2} \gg 4 m F_{r} / r q^{2},(6)$ becomes

$$
v_{\theta, d r i f t} \cong \frac{q r_{0}}{2 m}\left|B_{z e x t}\right|\left[ \pm\left(1+\frac{2 m F_{r}}{r q^{2} B_{z e x t}^{2}}\right)-\operatorname{sign}\left(B_{z e x t}\right)\right]_{r_{0}}
$$

This reduces to the conventional expression for drift velocity $\vec{v}_{\text {drift }}=\vec{F} \times \vec{B} / q B^{2}$ only when one chooses the sign of the radical to be equal to $\operatorname{sign}\left(B_{\text {zext }}\right)$. With this choice, it is easily verified that the drift current $q v_{\theta, \text { drift }}$ of the charged particle given by (6) is paramagnetic in the sense that the magnetic field produced by it is in the direction of the external axial magnetic field regardless of the sign of the charge. At the point $F_{r}=0$, the right hand side of (2)no longer has the form of a potential well leading to unbounded orbits; hence $v_{\theta, d r i f t}=0$ when $F_{r}=0$.

In the presence of $n_{e}$ electrons $\left(q_{e}=-e\right)$ per unit volume experiencing the same force per particle, there would an azimuthal current density leading to an axial magnetic field:

$$
-\frac{\partial B_{z}}{\partial r}=\mu_{0} J_{\theta}=\mu_{0} n_{e} q_{e} v_{\theta, e}=\frac{\mu_{0} n_{e} e^{2} r}{2 m_{e}}\left[\sqrt{B_{z}{ }^{2}+\frac{4 m_{e} F_{r}}{r e^{2}}}-B_{z}\right]
$$

This equation admits solutions for $B_{z}$ which pass through $B_{z}=0$. For $B_{z}{ }^{2}>>\frac{4 m_{e} F_{r}}{r e^{2}}, B_{z}>0$, it gives

$$
\frac{\partial}{\partial r} \frac{B_{z}^{2}}{2 \mu_{0}}=-n_{e} F_{r}
$$

For $B_{z}<0$, equation 8 gives a solution which rapidly grows on the scale length of $c^{2} / r_{s} \omega_{p e}^{2}$ where $r_{s}$ is the radius at $B_{z}=0$. This clearly unphysical situation can be avoided 
by stipulating as a model assumption that the conditions $\partial B_{z} / \partial r=0$ (which implies $v_{\theta}=0$ and hence $\left.F_{r}=0\right)$ and $B_{z}=0$ occur at coincident points.

The derivation of this expression for the drift velocity makes no reference to any continuum model of the plasma. However, in the precursor plasma region, the average force experienced by an electron would be the total pressure gradient ${ }^{28}-\hat{r} \partial p / \partial r$ divided by electron density $n_{e}$. The foregoing discussion then implies that in the PP region, $\partial p / \partial r=0$ at the point $B_{z}=0$. This model assumption is compatible with all the available experimental evidence: there is no direct measurement of pressure and there does exist alocal maximum of the luminosity signal (which, like the pressure, is an increasing function of both density and temperature) in fig $4 \mathrm{a}$ of Krauz et al. in Ref 22 coinciding with the $B_{z}=0$ point. With both the axial magnetic field and its derivative being zero at the point of maximum pressure, equation (9) gives upon integration,

$$
B_{z} \simeq+\sqrt{2 \mu_{0}\left(p_{\max }-p(r)\right)}
$$

Assuming a density $\sim 1.27 \times 10^{17} \mathrm{~cm}^{-3}$ (corresponding to fill density of $\mathrm{D}_{2}$ at 1.8 torr) and a temperature $\sim 0.1 \mathrm{eV}$ in the PP region for fig $4 \mathrm{a}$ of Ref. 22 , which borders the nonluminous neutral gas, (10) predicts $B_{z} \sim+\sqrt{2 \mu_{0}(2 n k T)} \sim 1 \mathrm{kG}$, in reasonable agreement with both the observed order of magnitude and polarity.

According to the above referred fig $4 \mathrm{a}$, the point of $B_{z}=0$ is immediately followed by the azimuthal magnetic field signal, showing presence of the current carrying high conductivity plasma region, which is azimuthally continuous. When magnetic flux gets created by the above described mechanism within this annular conducting plasma, Lenz's law would create an azimuthal electric field driving azimuthal current in the annular conductor tending to create axial magnetic field of opposite polarity. The charged particle drift analysis derived above is sufficiently general to apply to this region with the difference that the ambientmagnetic field generated as a result of Lenz's Law would now be in $-\hat{z}$ direction and the net radial force per particle would be given by

$$
-F_{r} \hat{r}=-\hat{r} \frac{1}{n_{e}}\left(J_{z} B_{\theta}+\frac{\partial p}{\partial r}\right)
$$

The axial magnetic field would be described by equation (8) together with (11), with the $z$ axis now understood to be of reversed polarity. Taking into account the reversed polarity, (9) and (11) yield for the axial magnetic field in the PCS

$$
B_{z}=-\sqrt{2 \mu_{0} \int_{r}^{r_{s}} d r\left(J_{z} B_{\theta}+\frac{\partial p}{\partial r}\right)}
$$

The paramagnetic drift current model is seen to be compatible with experimental observations including those about radial confinement of copper plasma plume in 
experiments at Lebedev Institute ${ }^{17,18}$, since (10) predicts a non-zero, constant $B_{z}$ at $r=0$, which would influence the plasma plume but would not be detected by a magnetic probe, which provides only differential measurements.

\section{Implications and possibilities for experimental investigations}

Evolution of axial magnetic field would be necessarily accompanied by an azimuthal electric field which can accelerate ions, radially confined by azimuthally symmetric implosive forces, along an infinite path in the azimuthal direction. The maximum velocity resulting from such acceleration, which is independent of any instability (as in the case of PLAMYA installation ${ }^{30}$ which had no instability), is given by the product of charge-tomass ratio and the azimuthal component of vector potential. It can be verified that ion kinetic energy in the $\mathrm{MeV}$ range may easily be produced by such acceleration for ballpark values of DPF parameters although it would be limited in practice to smaller values because of collisions. Since $B_{z}$ is proportional to the current in the order of magnitude according to (12), the ion energy should be linearly dependent on the square of current; there is experimental evidence ${ }^{31}$ to this effect. This implies that nuclear reactions with high threshold energies, such as $\mathrm{p}-{ }^{11} \mathrm{~B}$ fusion or ${ }^{16} \mathrm{O}\left({ }^{3} \mathrm{He}, \mathrm{p}\right){ }^{18} \mathrm{~F}$ can be made to occur efficiently near the peak of their reaction cross-sections, utilizing energetic ions automatically produced and confined within the plasma by this process. It is worth

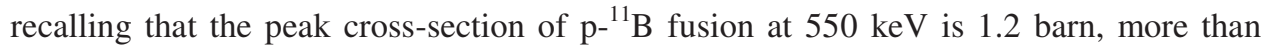
$1 / 5^{\text {th }}$ the peak cross-section of D-T fusion reaction; occurrence of abundant ions in this energy range is already indicated ${ }^{10,11,12}$ by experimental data. Commercial utilization of $D P F$ for such reactions should then be much more readily feasible than is currently understood.

It should therefore be important to validate /refute this mechanism by experiments. A direct test of (10) can be made by placing lens-coupled optical fiber probes at off-axis positions in the anode, which would pick up light from a well-defined volume element of limited size in front of the anode. This can be analyzed using a time-resolving visible spectrograph $^{32}$. Before the arrival of the plasma current sheath in the field of view, this probe would sample visible emission from the relatively cold, partially photo-ionized precursor plasma, which would contain space and time resolved information about electron density, temperature and local magnetic field from line profile, line intensity ratio and Zeeman splitting measurements respectively on hydrogen and impurity lines. Absolute calibration of the detection system can yield neutral gas density. There would be no interference from the azimuthal magnetic field, which would not be in the field of view, facilitating unambiguous interpretation of Zeeman data, from which a fully spectroscopic quantitative test of (10) could be realized. A unique benefit of the proposed test is that all measured quantities would pertain to the same volume element and to the same instant in time, requiring no spatial unfolding or temporal corrections unlike conventional line-of-sight integrated spectroscopy.

One signature of this mechanism is emission of flux of axial magnetic field, which can be detected outside the cathode ${ }^{20}$. A conclusive test of this mechanism would look for 
switching of the polarity of the emitted axial magnetic flux by placing the DPF in a Helmholtz coil producing 1G magnetic field of reversible polarity. Both positive and negative results of this experiment would be significant: in case the polarity of the emitted flux is switched by the external Helmholtz coil, it would demonstrate behavior analogous to ferromagnetism in a plasma. If it does not switch, it leaves the question of origin of the observed fixed polarity of the solenoidal state of DPF begging for an answer once again, ruling out ambient magnetic field as the vector field responsible.

\section{DPF as a technology platform for industrial applications}

Appreciation $^{33,34}$ of possible commercial applications of DPF inevitably has to face up to the current hardware reality, where laboratory installations are built in-house from available capacitors, switches and cables and are quite prone to frequent malfunction - a reality not conducive to industrial-scale investments in development of DPF as a technology platform. This reality is rooted in the historical fact that low inductance capacitors were originally developed for pinch research in the 1950's and were optimized for connections to switches and cables for incorporation into large capacitor banks. Later developments concentrated on Marx banks and electromagnetic guns as major applications. Designs of capacitors and switches optimized for use with DPF devices are technically possible but are not a current funding priority.

Industrial-scale investment in development of industrial-quality DPF hardware calls for some pre-requisites. Foremost is a well-founded basis for the design using experimentally validated design tools and procedures and first-principles optimization criteria (not entirely rooted in empirical thumb-rules) together with possibility of automated parameter search for optimum configurations to avoid advisor bias. This search for a globally optimized "best possible" design needs to maximize quantitative performance criteria per unit of stored energy, using the least number of independent components to minimize chances of failure - a design which must be adaptable to diverse application requirements and which should have a built-in scalability so that larger or smaller versions can be chosen to give the best rate-of-return on investment. Also important are innovations, which substantially deviate from the current state-of-art and improve the DPF operation in significant ways, so that the investment can be protected with patents. Such development should ultimately result in custom-designed, ready-touse DPF facilities, ruggedly engineered with minimum maintenance requirements, with integrated first-principles design of capacitors, switches and the plasma device, with engineered flexibility for empirical optimization for particular requirements, with additional non-standard modes of operation to provide extra degrees of freedom.

Construction of an appropriate design tool then becomes the first important step in this direction. Towards this end, recently revisited Gratton-Vargas model ${ }^{35}$ can currently calculate optimality parameters of designs at the rate of 15000 designs a day with considerable scope for further improvements in speed. This model maps the 10 parameters of a DPF installation - capacitance, inductance, resistance, voltage, anode radius, anode length, insulator radius, insulator length and gas pressure - on to 7 
dimensionless model parameters, out of which 5 are relevant for optimization. This circumstance enables generation of a database of a limited set of optimality properties (such as average power transfer efficiency, electromagnetic work, energy per particle, fraction of energy dissipated in circuit resistance etc.) of Mather type DPF devices in a practically relevant range of parameters. Examination of technical logic reveals significant opportunities for practical realization of globally-optimized industrial quality integrated DPF hardware in not-too-distant future, using existing manufacturing technology and relatively modest resources, designed using automated search algorithms over such compilation of optimality properties.

\section{Conclusion}

To summarize, in its role as a question in search of answers, DPF is poised to be harbinger of new physics; as a technology in search of applications, it serves as a beacon for new opportunities in commercial workspace for those bold enough to venture.

\section{References}

1. V. V. Vikhrev and V. D. Korolev, Plasma Physics Reports, 33356, (2007).

2. G. Bockle, J. Ehrhardt, P. Kirchesch, et al, Plasma Phys. Cont Fusion, 34, 801, (1992).

3. M. J. Bernstein, D. A. Mescan, and H. L. L. Van Passen, Phys. Fluids12, 2193 (1969).

4. J. H. Lee, L. P. Shomo, M. D. Williams, and H. Hermansdorfer, Phys. Fluids 14 2217(1971).

5. P. Kubes, et al, IEEE Trans. Plasma Science, 34 2349(2006).

6. Ch. Maisonnieret al, Plasma Phys. Cont. Nucl. Fusion, 1 523(1971).

7. M. J. Bernstein and G. G. ComisarPhys. Fluids15, 700(1972).

8. H. Schmidtet alPhysicaScripta. 66, 168,(2002)

9. M. V. Roshan, et al, Physics Of Plasmas 16 074506-1 (2009).

10. M.M. Milanese, J.O. Pouzo, Nucl. Fusion18, 533, (1978).

11. H. Conrads, P. Cloth, M., Demmeler and R. Hecker, Phys. Fluids, 15, 209 (1972).

12. A. Bernard et al.,Phys. Fluids, 18180, (1975).

13. J.P. Rager, "Progresses on Plasma Focus research at Frascati", Invited paper at the 10th European Conference on Plasma Physics and CTR", Frascati Report 81.43/cc;

14. U. Jaeger, Ph.D. Thesis, IPF-86-1, 1986,

15. S. K. H. AuluckPhysics of. Plasmas, 18, 032508(2011).

16. U. Jäger and H. Herold, Nucl. Fus. 27407 (1987).

17. V.A. Gribkov, et al, http://jphyscol.journaldephysique.org/articles/jphyscol/pdf/1979/07/physcol197940C7369.pdf

18. Gribkov V A, et al. Proc. PN Lebedev Physical Institute, Allerton Press, N-Y, 127 32, 1980.

19. Ehrhardt J, Fischfeld G, Kirchesch P, Hubner K, Rager J P 1981 Experimental evidence for $B z$-field and microturbulences in the Frascati PF facility,10th Europ. Conf. on Contr. Fusion and Plasma Phys., Moscow, Post-deadline paper, - 4 p.

20. S.K.H. Auluck, IEEETPS41437 (2013)

21. S. K. H. Auluck, IEEETPS25 37(1997).

22. V. I. Krauz, et alEurPhy. Lett, $\mathbf{9 8}$ 45001(2012).

23. P Kubes, et alPlasma Phys. Control. Fusion54 105023(2012)

24. P Kubes, et al, Plasma Phys. Control. Fusion55 035011 (2013) 
25. http://www.ngdc.noaa.gov/geomag-web/

26. S.K.H. Auluck,Phys. of Plasmas, 94488 (2002)

27. Vladimir I. Kolobov, Phys. of Plasmas 20101610 (2013).

28. V. Rozhansky, Phys. of Plasmas 20101614 (2013).

29. S. K. H. Auluck, J. Plasma Phys. 32 349(1984)

30. V.A.Bahilov et al. Proc. 4th Intl. Workshop on Plasma Focus and Z-pinches, Warsaw, (1985)

31. Eric J. Lerner et al. Nukleonika 57205 (2012)

32. F. J. Goldin, B. T. Meehan, E. C. Hagen, and P. R. Wilkins, Rev. Sci. Inst. 81, 10E531 (2010).

33. R.S Rawat, IEEETPS, 41701 (2013).

34. V.A Gribkov, This conference

35. S K H Auluck, Phys. of Plasmas, 20112501 (2013). 